\title{
基于光致变色的联狮满烯二酮衍生物的新型光调控磁性的 分子基磁体的合成及性能
}

\author{
陈勇 $*, a$ 孟继本 $b$ \\ ( ${ }^{a}$ 安阳师范学院化学化工学院 安阳 455000) \\ ( ${ }^{b}$ 南开大学化学学院 天津 300071)
}

\begin{abstract}
摘要 分子基磁性材料, 可用于信息存储、磁成像、光磁开关等. 因此, 分子基磁体设计与合成一直是当今科学研究的 热门课题. 受分子基磁体的启发, 本研究合成了新型光调控磁性的分子基磁体 $\square \square$ 含有 2-硝基丙烷自由基和 2,2,6,6-四 甲基-1-氧基哌啶(TEMPO)自由基的联䒢满烯二酮衍生物 4. 化合物 4 的合成方法为，以光致变色的 7,7'-二甲基-3,3'-二 乙基-3,3'-二羟基-2,2'-二狮叉基-1,1'-二酮(1)为起始原料, 经过溴代反应，得到 7,7'-二(溴代甲基)-3,3'-二乙基-3,3'-二羟基 -2,2' -二狮叉基-1,1'-二酮(2). 化合物 2 和 2-硝基丙烷在乙醇钠的乙醇溶液中反应得到含有 2-硝基丙烷自由基和醛基的 联䒢满烯二酮衍生物 3. 根据 ${ }^{1} \mathrm{H}$ NMR, MS 和元素分析对化合物 3 的结构分析表明, 2-硝基丙烷基团和化合物 3 苯环上 的亚甲基相连. 对化合物 3 的 ${ }^{1} \mathrm{H} \mathrm{NMR}$ 和 ESR 波谱进一步的分析表明 2-硝基丙烷基团上存在单电子自旋. 在化合物 3 的基础上, 稳定的氮氧自由基 TEMPO 被成功地引入光致变色的联狮满烯二酮衍生物的苯环上得化合物 4. 对化合物 2,3 和 4 光照前后的性质变化进行了研究. 固态化合物 2,3 和 4 光照下皆可发生光致变色; 固态化合物 2 光照后可测 得 ESR 波谱; 固态化合物 3 和 4 光照前后皆可测得 ESR 波谱, 且光照前后 ESR 波谱发生变化. 磁化率测试表明, 固态 化合物 4 光照前后皆具有反铁磁性, 且光照后反铁磁相互作用增强. 测试结果表明固态化合物 $\mathbf{3}$ 和 4 光照后有两个或 三个自旋中心, 分别是 2-硝基丙烷或 TEMPO 自由基以及光照产生的来自于联狮满烯二酮的羰基上的自由基. 固态化 合物 4 的颜色和磁性可用光调控.
\end{abstract}

关键词 联䒢满烯二酮; 2-硝基丙烷自由基; 光控磁性; 光致变色; 分子基磁体

\section{Synthesis and Properties of Novel Photomodulation Magnetic Molecular-Based Magnet Based on Photochromic Biindenylidenedione Derivative}

\author{
Chen, Yong*,a Meng, Jiben ${ }^{b}$ \\ ( ${ }^{a}$ Department of Chemistry, Anyang Normal University, Anyang 455000) \\ ( ${ }^{b}$ Department of Chemistry, Nankai Univeristy, Tianjin 300071)
}

\begin{abstract}
Molecular-based magnetic material can be used for information storage, magnetic imaging, photo-magnetic switching and so on. So the design and synthesis of the molecular-based magnet is a focus of contemporary science research. Sparked by the strategy of molecular magnets, the novel photomodulation magnetic molecular-based magnet photochromic biindenylidenedione derivative with 2-nitropropane radical and 2,2,6,6-tetramethyl-piperidinyloxy (TEMPO) radical (4) was synthesized. The synthetic method of compound $\mathbf{4}$ is that starting from photochromic biindenylidenedione derivative 7,7'-dimethyl-[2,2'-bi-1H-indene]-3,3'-diethyl-3,3'-di-hydroxy-1,1'-dione (1), via bromination reaction, 7,7'-bis(bromomethyl)[2,2'-bi-1H-indene]-3,3'-diethyl-3,3'-dihydroxy-1,1'-dione (2) was obtained. Sodium ethoxide, 2-nitropropane and compound 2 were dissolved in absolute ethanol reaction to obtain biindenylidenedione derivative with 2-nitropropane radical and formyl 3. The structure of compound 3 was confirmed by ${ }^{1} \mathrm{H}$ NMR, MS and element analysis. The results indicated that 2-nitropropane group bonds with one methylene on the benzene ring of the compound 3. In further analysis to ${ }^{1} \mathrm{H}$ NMR and electron spin-resonace (ESR) spectra of compound 3 demonstrated that single electron spin exists in 2-nitropropane group. On the basis compound 3, stable nitroxide radical TEMPO was successfully introduced into the benzene rings of photochromic biindeny-
\end{abstract}

* E-mail: chenqiaoyong@aliyun.com

Received February 26, 2016; revised April 11, 2016; published online April 20, 2016. 
lidenedione derivative to obtain compound $\mathbf{4}$. The properties of compounds $\mathbf{2}, \mathbf{3}$ and $\mathbf{4}$ were investigated. The testing results display that the solid compounds $\mathbf{2} \sim \mathbf{4}$ possess photochromic property. ESR spectrum was found when irradiated solid compound 2. ESR spectrum was found and changed before and after photoirradiation in solid state of compound $\mathbf{3}$ and $\mathbf{4}$. The magnetic susceptibility measurement shows that the antiferromagnetic interaction for before and after photoirradiation in solid state of compound 4, and the antiferromagnetic interaction becomes stronger after photoirradiation. These testing results demonstrated that after photoirradiation in solid state of compound $\mathbf{3}$ and $\mathbf{4}$, there are two or three kinds of spin centres after photoirradiation: one is 2-nitropropane or TEMPO radical, and another is photo-generated radical of two indanione moieties. The color and magnetic of the solid compound $\mathbf{4}$ can be photomodulated.

Keywords biindenylidenedione; 2-nitropropane radical; photomodulation magnetism; photochromism; molecular-based magnet

磁性材料和光功能材料是材料科学研究的两个主 要领域. 在磁性材料中, 由分子基磁体构成的磁性材料, 尤其是和分子的其它性质结合的分子基磁性材料，可用 于信息存储、磁成像、光磁开关等, 因此分子基磁体设 计与合成一直是当今科学研究的热门课题 ${ }^{[1]}$. 在合成分 子基磁性材料的方法中, 纯有机自由基作为自旋源起着 重要作用. 如氮氧自由基 2,2,6,6-四甲基-1-氧基哌啶 (TEMPO)自由基和 2-取代-4,4,5,5-四甲基-1-氧基-3-氧 代咪唑啉自由基等, 被广泛应用于分子基磁性材料的合 成 ${ }^{[2]}$. 在光功能材料中, 有机光致变色化合物由于其可 作为光信息储存材料、光装饰材料以及用于防伪识别技 术等, 而引起科研工作者的研究兴趣 ${ }^{[3]}$. 在对有机光致 变色分子和分子基磁体进行广泛而深入的研究基础上, 广大科研工作者也在寻求把二者结合起来的途径, 即设 计合成把光致变色结构单元和磁性结构单元组合在一 起的分子基材料, 在这种分子基材料中通过光致变色结 构单元的光诱导作用可引起分子结构或分子中电子分 布的变化, 从而达到合成对光响应的分子基磁体的目 的 ${ }^{[4]}$. Irie 和 Matsuda 在这方面做了较为深入的研究, 他 们成功地把稳定的氮氧自由基嫁接到光致变色的二芳 基乙烯类化合物分子的母体中, 在溶液中这种分子通过 不同波长的光照, 其分子中二芳基乙烯的结构可逆的发 生变化, 从而导致其颜色发生可逆的变化, 同时分子两 端的磁性部分(氮氧自由基)的相互作用也发生变化, 导 致分子的磁性在光照前后发生变化, 在此过程中二芳基 乙烯结构起到了一种光可调控分子的颜色和磁性的光 调控单元的作用 ${ }^{[5]}$. 以往的研究表明, 联狮满二羟基烯 二酮衍生物是一类独特的光致变色有机化合物, 其固态 受到光照后, 分子中电子排布发生变化, 导致其分子中 $\pi$ 电子离域范围增大, 造成其光照前后的紫外-可见吸 收光谱发生显著变化, 因而也导致其光照前后的颜色发 生变化, 即光致变色. 同时由于分子中电子排布的变化, 导致其分子中两个羰基的氧上产生自由基. 其光致变色 和自由基产生机理如 Eq. 1 所示 ${ }^{[6]}$.

参考文献 [5]的研究思路, 设想把氮氧自由基引入 光致变色的联茆满二着基烯二酮衍生物分子两端的苯

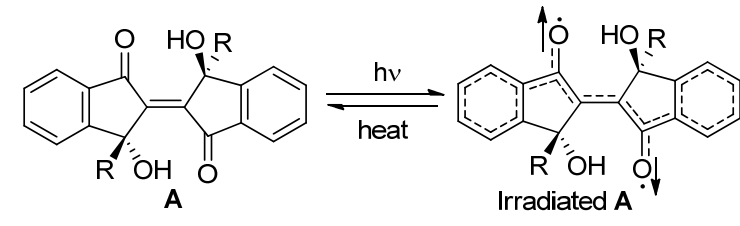

环上，所得到的化合物在固态光照后，分子中的联狮满 二羟基烯二酮结构单元的 $\pi$ 电子排布发生变化，导致其 分子中该结构单元的 $\pi$ 电子离域范围增大，同时在两个 羰基的氧上产生自由基. 因而得到的此种化合物不仅光 照前后的颜色发生变化，即具有光致变色性质，而且其 光照前分子中只有一种自旋源(Eq. 2), 来自氮氧自由基; 在光照后具有两种自旋源(Eq. 2, Irradiated B), 一种是 连接于联狮满二羟基烯二酮衍生物的苯环上的氮氧自 由基，另一种为光照产生的自由基，位于分子中的二羰 基部分. 这种化合物光照后分子中的两种自旋源进行相 互作用，导致其分子的磁性也发生变化，即其分子的磁 性可以进行光调控, 是一种光可调控颜色和磁性的分子 基磁体. 联䒢满二羟基烯二酮结构单元的这种独特性 质，使其可作为影响分子内或分子间自旋相互作用的光 调控单元.
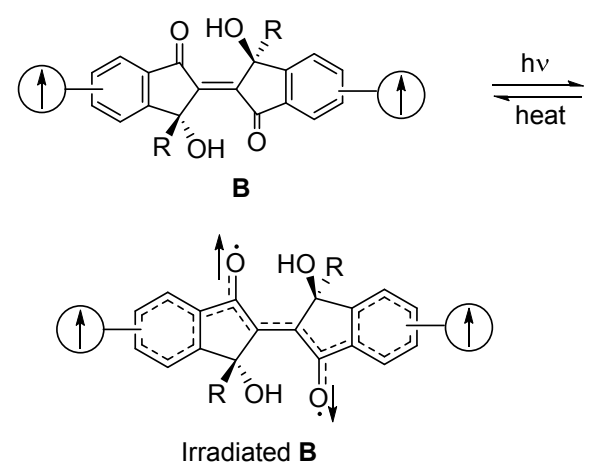

在以前研究工作的基础上, 为了进一步研究这种新 型光调控的分子基磁体，设计了一条以化合物 $\mathbf{1}$ 为起始 原料，经中间体二醛 $\mathbf{X}$ 合成目标化合物 $\mathbf{Y}$ 的合成路线 (Scheme 1). 但是实验的结果并没有得到期望的关键中 间体二醛 $\mathbf{X}$, 而是得到了一种预料之外的化合物 $\mathbf{3}$, 最 终产物及其中间体的实际合成路线如 Scheme 2 所示. 
<smiles>C=CC=CC</smiles><smiles>[X]C1(O)C(=C2C(=O)c3cc(C=O)ccc3[C@]2(O)CC)C(=O)c2cc(C=O)ccc21</smiles><smiles>[X][C@@H]1C(=C2C(=O)c3cc(C=NC4CC(C)(C)N(O)C(C)(C)C4)ccc3[C@@]2(O)CC)C(=O)c2cc(C=NC3CC(C)(C)N([O])C(C)(C)C3)ccc21</smiles>

图式 1 设计的目标化合物的合成路线

Scheme 1 Plot synthesis route of title compound<smiles>CC[C@]1(O)C(=C2C(=O)c3cc(C)ccc3C2(O)O)C(=O)c2cc(C)ccc21</smiles><smiles>CC[C@@]1(O)C(=C2C(=O)c3cc(CBr)ccc3[C@]2(O)CC)C(=O)c2cc(CBr)ccc21</smiles><smiles>CC[C@]1(O)C(=C2C(=O)c3cc(C=O)ccc3C2(O)O)C(=O)c2cc(CON([O])C(C)C)ccc21</smiles><smiles>CC[C@@]1(O)C(=C2C(=O)c3cc(CON(C)C(C)C)ccc3C2(O)O)C(=O)c2cc(C=NC3CC(C)(C)N([O])C(C)(C)C3)ccc21</smiles>

Reagents and conditions: (a) NBS/ $\mathrm{C}_{6} \mathrm{H}_{6}, 41 \%$; (b) $\left(\mathrm{CH}_{3}\right)_{2} \mathrm{CHNO}_{2}$, $\mathrm{C}_{2} \mathrm{H}_{5} \mathrm{ONa} / \mathrm{C}_{2} \mathrm{H}_{5} \mathrm{OH}, 32 \%$; (c) TEMPO/C ${ }_{6} \mathrm{H}_{6}, 23 \%$

图式 2 最终产物的实际合成路线

Scheme 2 Actual synthetic route of title compound

\section{1 结果与讨论}

\section{1 中间体 3 的分子结构分析及存在新自旋源的推理}

为了达成研究目标, 设想按照 (Scheme 1) 的合成路 线, 以化合物 1 为起始原料, 首先合成关键中间体二醛 $\mathbf{X}$. 为了合成中间体二醛 $\mathbf{X}$, 查阅了大量文献, 发现通 过芳甲基化合物合成芳香醛有多种方法 ${ }^{[7]}$. 以化合物 $\mathbf{1}$ 为反应物, 参考前述文献[7]记载的多种实验方法, 均未
得到想要的中间体二醛 $\mathbf{X}$. 在查阅文献的过程中，发现 文献[8]利用芳香环上含有溴代甲基的芳香化合物经化 学反应可制得芳香醛. 参考文献[8]的方法, 用化合物 $\mathbf{1}$ 先把其苯环上的甲基溴化，生成含溴代甲基的产物，接 着用文献[8]的方法进行醛的合成较为成功. 但对中间 体醛的分子结构进行分析测试的结果表明，其分子结构 式并不是想要合成的中间体二醛 $\mathbf{X}$, 而是一种预料之外 的产物单醛化合物 $\mathbf{3}$, 如 Scheme 2 所示. 利用化合物 3 再与含有稳定氮氧自由基的 4-amino-TEMPO 反应生成 最终产物 4.

为了便于对化合物 3 的分子结构进行分析确证，把 其前体化合物 1 和 2 的 ${ }^{1} \mathrm{H}$ NMR 图谱一并分析，通过分 析化合物 1 3 的 ${ }^{1} \mathrm{H}$ NMR 图谱的变化, 发现它们分子 结构的变化.

从 Scheme 2 所示的合成路线可知，从化合物 1 到化 合物 3 所发生的两步反应，反应发生的位置都位于分子 中 7,7'位的基团上，主要分析该位置基团上 ${ }^{1} \mathrm{H}$ NMR 图 谱的变化.

化合物 2 的 ${ }^{1} \mathrm{H}$ NMR 图谱(图 1)和化合物 $\mathbf{1}$ 的 ${ }^{1} \mathrm{H}$ NMR 图谱对比发现，化合物 1 原在 $\delta 2.48$ 处 7,7'- $\left(\mathrm{CH}_{3}\right)_{2}$ 的吸收峰在化合物 $\mathbf{1}$ 经溴代生成化合物 $\mathbf{2}$ 后此吸收峰则 完全消失, 化合物 2 在 $\delta 4.58(\mathrm{~s}, 4 \mathrm{H})$ 处新出现一个吸收 峰, 应是和苯环直接相连的 7,7'-( $\left(\mathrm{CH}_{2} \mathrm{Br}\right)_{2}$ 的吸收峰. 化 合物 1 和 2 分子中其余氢 ${ }^{1} \mathrm{H}$ NMR 图谱吸收峰的 $\delta$ 值基 本保持不变.

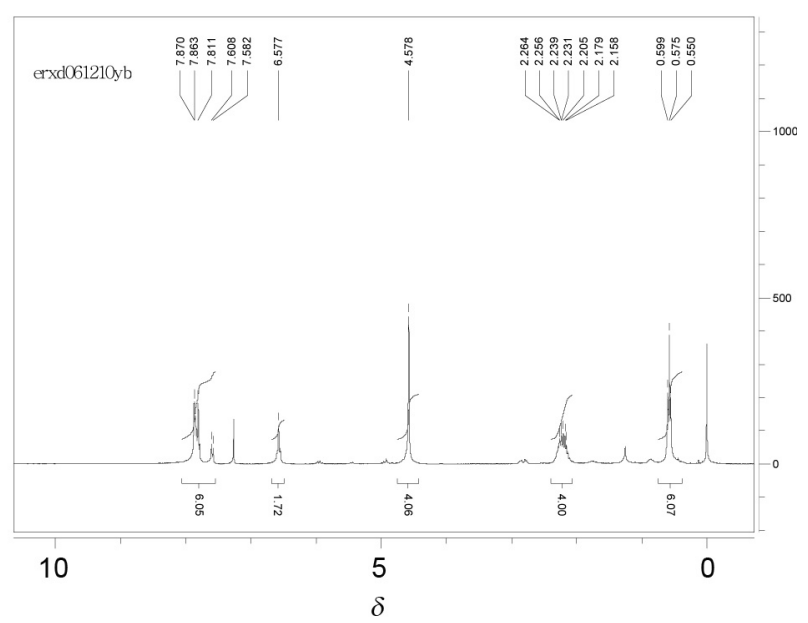

图 1 化合物 2 的 ${ }^{1} \mathrm{H} \mathrm{NMR}$

Figure $1{ }^{1} \mathrm{H}$ NMR of compound 2

对化合物 3 的分子结构进行分析测试的结果表明, 其质谱为 ESI $m / z: 476.4[\mathrm{M}-\mathrm{H}]^{+}$和要合成的二醛 $\mathbf{X}$ 的 分子量完全不符 $\left(\mathbf{X}\right.$ 分子量为 404.1). 化合物 3 的 ${ }^{1} \mathrm{H}$ NMR 图谱(图 2), 共出现 12 个吸收峰, 各组峰的积分比 为 $6: 6: 4: 2: 1: 1: 1: 1: 1: 1: 2: 1$. 


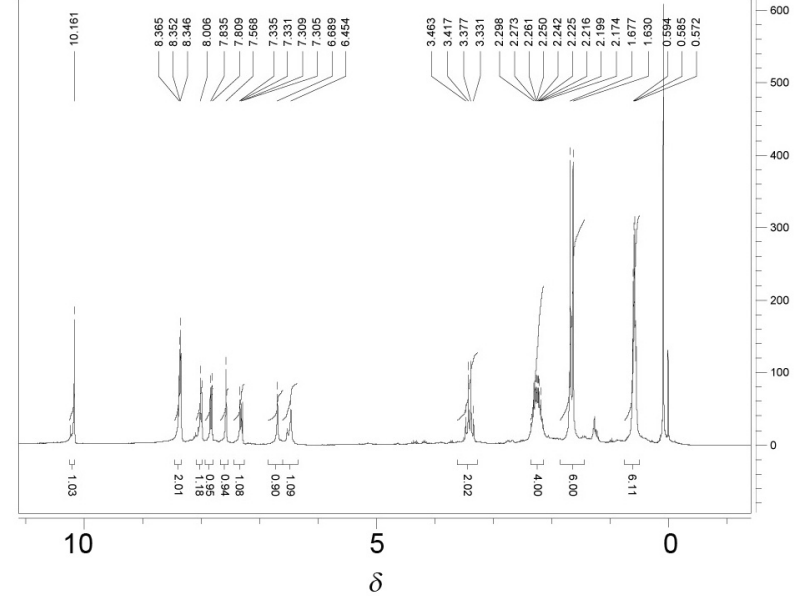

图 2 化合物 3 的 ${ }^{1} \mathrm{H}$ NMR

Figure $2{ }^{1} \mathrm{H}$ NMR of compound 3

化合物 $\mathbf{3}$ 的 ${ }^{1} \mathrm{H}$ NMR 图谱和化合物 $\mathbf{2}$ 的 ${ }^{1} \mathrm{H}$ NMR 图 谱比较, 除 3,3'- $\left(\mathrm{CH}_{2} \mathrm{CH}_{3}\right)_{2}$ 吸收峰的 $\delta$ 值基本保持不变 外, 其余氢的吸收峰发生了显著变化, 且出现了多个新 的吸收峰. 原在化合物 2 中 $\delta 4.58(\mathrm{~s}, 4 \mathrm{H})$ 处, 7'- $\left(\mathrm{CH}_{2} \mathrm{Br}\right)_{2}$ 的吸收峰, 当化合物 2 反应转化为化合物 $\mathbf{3}$ 后, 此吸收 峰完全消失. 化合物 3 的 ${ }^{1} \mathrm{H}$ NMR 图谱在 $\delta 1.66(\mathrm{~d}, J=$ $14.1 \mathrm{~Hz}, 6 \mathrm{H}), 2.17 \sim 2.30(\mathrm{~m}, 4 \mathrm{H}), 10.16(\mathrm{~s}, 1 \mathrm{H})$ 处出现了 新的吸收峰. 根据常见官能团的 ${ }^{1} \mathrm{H}$ NMR 图谱 $\delta$ 值推测, 在 $\delta 10.16$ 处的吸收峰应为 $\mathrm{CHO}$ 吸收峰, 按积分比为一 个氢, 说明化合物 $\mathbf{3}$ 中只存在一个 $\mathrm{CHO}$, 即化合物 $\mathbf{2}$ 中 7- $\mathrm{CH}_{2} \mathrm{Br}$ 转化为了 7-CHO. 而 $\delta 1.66(\mathrm{~d}, J=14.1 \mathrm{~Hz}, 6 \mathrm{H})$, $2.17 \sim 2.30(\mathrm{~m}, 4 \mathrm{H})$ 处的新吸收峰应该如何归属? $7^{\prime}-\mathrm{CH}_{2} \mathrm{Br}$ 转化为了何种基团?

根据文献[8]的反应历程 (Scheme 3), 可能是化合物 2 在反应后, 7'- $\mathrm{CH}_{2} \mathrm{Br}$ 和 2-硝基丙烷负离子结合后没有 完成 $\mathrm{CH}_{2} \mathrm{Br}$ 向 $\mathrm{CHO}$ 的转换, 而是形成了如图 3 所示的 分子片段. 化学位移 $\delta 1.66$ 处的 6 个氢应为硝基丙烷上 的两个 $\mathrm{CH}_{3}$, 化学位移 $\delta 3.33 \sim 3.46$ 处的 2 个氢为与苯 环相连的 $\mathrm{CH}_{2}$. 这两处的吸收峰如果无其它因素的影响 应是单吸收峰, 令人感到困惑的是为什么这两处的吸收 峰裂分为双重峰和四重峰, 而不是单峰呢? 可能的原因 是二硝基丙烷基团上的某些原子存在单电子, 而单电子 自旋的存在可引起相邻 $\mathrm{CH}_{3}$ 和 $\mathrm{CH}_{2}$ 的 ${ }^{1} \mathrm{H}$ NMR 图谱吸 收峰的分裂. 单电子自旋形成的可能机理如 Scheme 4 所示. 正如 Scheme 4 所提出的单电子自旋形成的机理, 化合物 2 中 7'- $\mathrm{CH}_{2} \mathrm{Br}$ 和 2-硝基丙烷负离子发生反应后 生成了如图 3 所示的分子片段. 在该分子片段中的 2-硝 基丙烷基团存在一个叔碳自由基和一个氮氧自由基, 正 是由于这两个自由基单电子自旋所引发的磁效应造成 了相邻的 $\mathrm{CH}_{3}$ 和 $\mathrm{CH}_{2}{ }^{1} \mathrm{H}$ NMR 图谱吸收峰的分裂. 检验 是否存在单电子自旋的最有效方法是做化合物的电子
自旋共振波谱(ESR 谱). 对化合物 3 在固态和溶液中分 别做过电子自旋共振波谱(ESR 谱)后, 发现化合物 3 无 论是在固态或是在溶液中都有明显的 ESR 信号出现(图 4). 说明在化合物 3 中 2-硝基丙烷基团上确实存在单电 子，即在化合物 3 中存在自旋中心(磁性源).

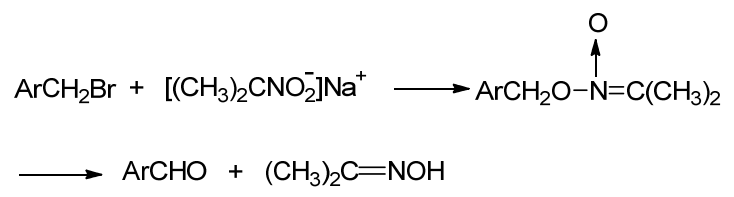

图式 3 反应历程

Scheme 3 Mechanism of reaction<smiles>CC(C)N(C)OCCO</smiles>

图 3 2-硝基丙烷自由基和亚甲基构成的分子片段

Figure 3 Molecular fragment that consist of 2-nitropropane radical and methylene
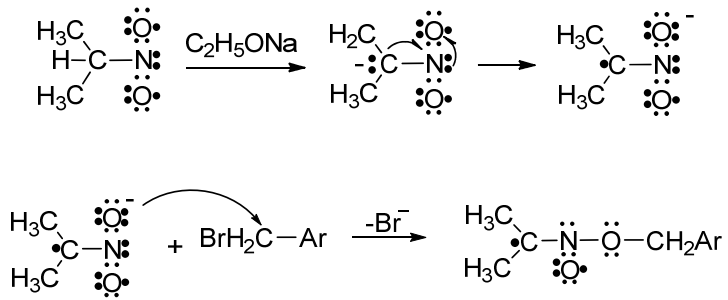

图式 4 推测的硝基丙烷引入联狮满烯二酮衍生物分子中以 及单电子自旋形成的机理

Scheme 4 Speculative mechanism of 2-nitropropane was introduced the molecule of biindenylidene derivative and formation single electron spin

在确定化合物 3 分子中存在 7-CHO 和 7'位(图 3)分 子片段后，就容易解释在化合物 2 中 $3,3^{\prime}-(\mathrm{OH})_{2}$ 的 ${ }^{1} \mathrm{H}$ NMR 图谱吸收峰为一个单峰 $\delta 6.58(\mathrm{~s}, 2 \mathrm{H})$, 因为 $3,3^{\prime}$ $(\mathrm{OH})_{2}$ 的化学环境完全相同(分子为对称结构), 即化学 等价，磁等价. 在化合物 3 中 $\mathrm{OH}$ 的 ${ }^{1} \mathrm{H}$ NMR 图谱吸收 峰变为两个单峰, 分别为 $\delta 6.69(\mathrm{~s}, 1 \mathrm{H}, 3-\mathrm{OH}), 6.45(\mathrm{~s}$, $\left.1 \mathrm{H}, 3^{\prime}-\mathrm{OH}\right)$, 因为化合物 3 中两个 $\mathrm{OH}$ 的化学环境不再相 同(不再是对称分子), 即化学不等价, 磁不等价. 在化 合物 2 中苯环上的氢 ${ }^{1} \mathrm{H}$ NMR 图谱吸收峰在 $\delta 7.58 \sim$ $7.87(\mathrm{~m}, 6 \mathrm{H})$ 处形成多重吸收峰. 而在化合物 3 中苯环上 的氢 ${ }^{1} \mathrm{H}$ NMR 图谱由于两个苯环上分别含有醛基和图 3 所示的分子片段, 受此基团的影响, 分裂为多个吸收峰. 含醛基的苯环氢的 ${ }^{1} \mathrm{H}$ NMR 图谱吸收峰为 $\delta 8.01(\mathrm{~s}, 1 \mathrm{H})$, $8.36(\mathrm{t}, J=2.9 \mathrm{~Hz}, 2 \mathrm{H})$, 含图 3 所示分子片段的苯环氢的 ${ }^{1} \mathrm{H}$ NMR 图谱吸收峰为 $\delta 7.31 \sim 7.34(\mathrm{~m}, 1 \mathrm{H}), 7.57(\mathrm{~s}$, $1 \mathrm{H}), 7.83(\mathrm{~d}, J=7.8 \mathrm{~Hz}, 1 \mathrm{H})$. 


\section{2 溶液中的紫外-可见吸收光谱}

化合物 $2 \sim 4$ 在二氯甲烷溶液中的紫外-可见吸收峰 和摩尔吸光系数如表 1 和图 4 所示. 化合物 2,3 各有两 个吸收峰, 分别在 $226.0,318.5 \mathrm{~nm}$ 和 $227.5,331.5 \mathrm{~nm}$. 化合物 2, 3 在短波带 $\left(\lambda_{\text {max }}=226.0,227.5 \mathrm{~nm}\right)$ 的最大吸收 峰比其在长波带 $\left(\lambda_{\text {max }}=318.5,331.5 \mathrm{~nm}\right)$ 的吸收强度要 大. 而化合物 4 仅有一个强吸收峰, 位于 $\lambda_{\text {max }}=234 \mathrm{~nm}$ 处, 其摩尔吸光系数在化合物 $2 \sim 4$ 中是最强的 $(\varepsilon=$ $32037 \mathrm{~L} \cdot \mathrm{mol}^{-1} \cdot \mathrm{cm}^{-1}$ ). 与化合物 $\mathbf{2 ,}, \mathbf{3}$ 比较, 化合物 $\mathbf{4}$ 在短 波带的吸收峰有一定的红移( $7 \sim 8 \mathrm{~nm})$. 化合物 4 在长波 带的吸收峰被淹没在其短波带的强吸收峰中，导致其形 成宽展的强吸收峰. 和以前的研究一样, 化合物 $\mathbf{2} \sim \mathbf{4}$ 在 二氯甲烷溶液中的颜色光照前后不发生颜色变化.

表 1 化合物 $2 \sim 4$ 在二氯甲烷溶液中的 UV-Vis 吸收波谱最大 吸收波长和摩尔吸光系数

Table 1 UV-Vis absorption maxima and coefficients of $\mathbf{2} \sim \mathbf{4}$ in $\mathrm{CH}_{2} \mathrm{Cl}_{2}$ solution

\begin{tabular}{clc}
\hline 化合物 & \multicolumn{1}{c}{$\lambda_{\max } / \mathrm{nm}\left[\varepsilon /\left({\left.\left.\mathrm{L} \cdot \mathrm{mol}^{-1} \cdot \mathrm{cm}^{-1}\right)\right]}^{c} c /\left(\mathrm{mol} \cdot \mathrm{L}^{-1}\right)\right.\right.$} \\
\hline $\mathbf{2}$ & $226.0(24116), 318.5(18075)$ & $2.0 \times 10^{-5}$ \\
$\mathbf{3}$ & $227.5(24338), 331.5(14823)$ & $1.9 \times 10^{-5}$ \\
$\mathbf{4}$ & $234(32037)$ & $2.3 \times 10^{-5}$ \\
\hline
\end{tabular}

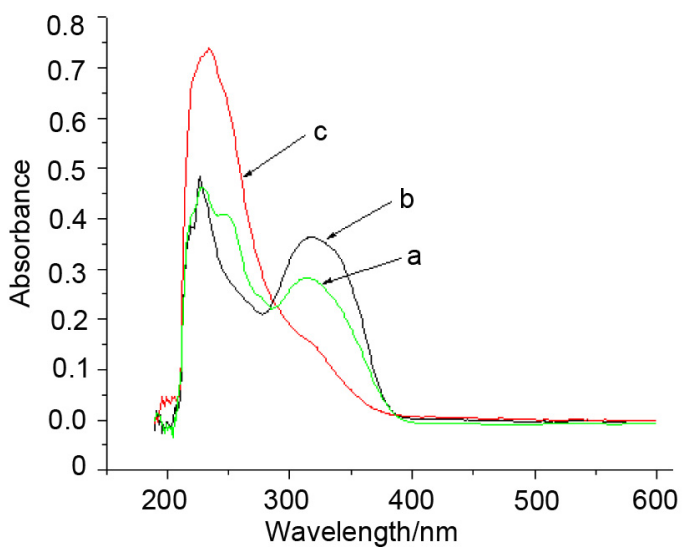

图 4 化合物 2 4 在二氯甲烷溶液中的 UV-Vis 光谱图

Figure 4 UV-Vis absorption spectra of compound $2 \sim 4$ in $\mathrm{CH}_{2} \mathrm{Cl}_{2}$ solution

$$
\text { (a) } 2 \text {, (b) } 3 \text {, (c) } 4
$$

\section{3 固态光致变色性质}

固态化合物 $2 \sim 4$ 在高压录灯照射下都具有明显的 光致变色性质. 光照前后的颜色变化见表 2 . 和以前的 研究 ${ }^{[6]}$ 类似, 化合物 $\mathbf{2} \sim \mathbf{4}$ 被高压录灯照射后, 其在 $500 \sim 800 \mathrm{~nm}$ 波长范围内的紫外-可见吸收有相当大的 增强(图 5). 化合物 2,3 光照后无论在短波带 $(350 \sim 450$ $\mathrm{nm})$ 还是在长波带 $(500 \sim 800 \mathrm{~nm})$ 其吸收强度都有所增 强, 而化合物 4 光照后在短波带吸收强度变弱(250 $350 \mathrm{~nm}$ ). 其光致变色机理以化合物 4 为例(Eq. 3).
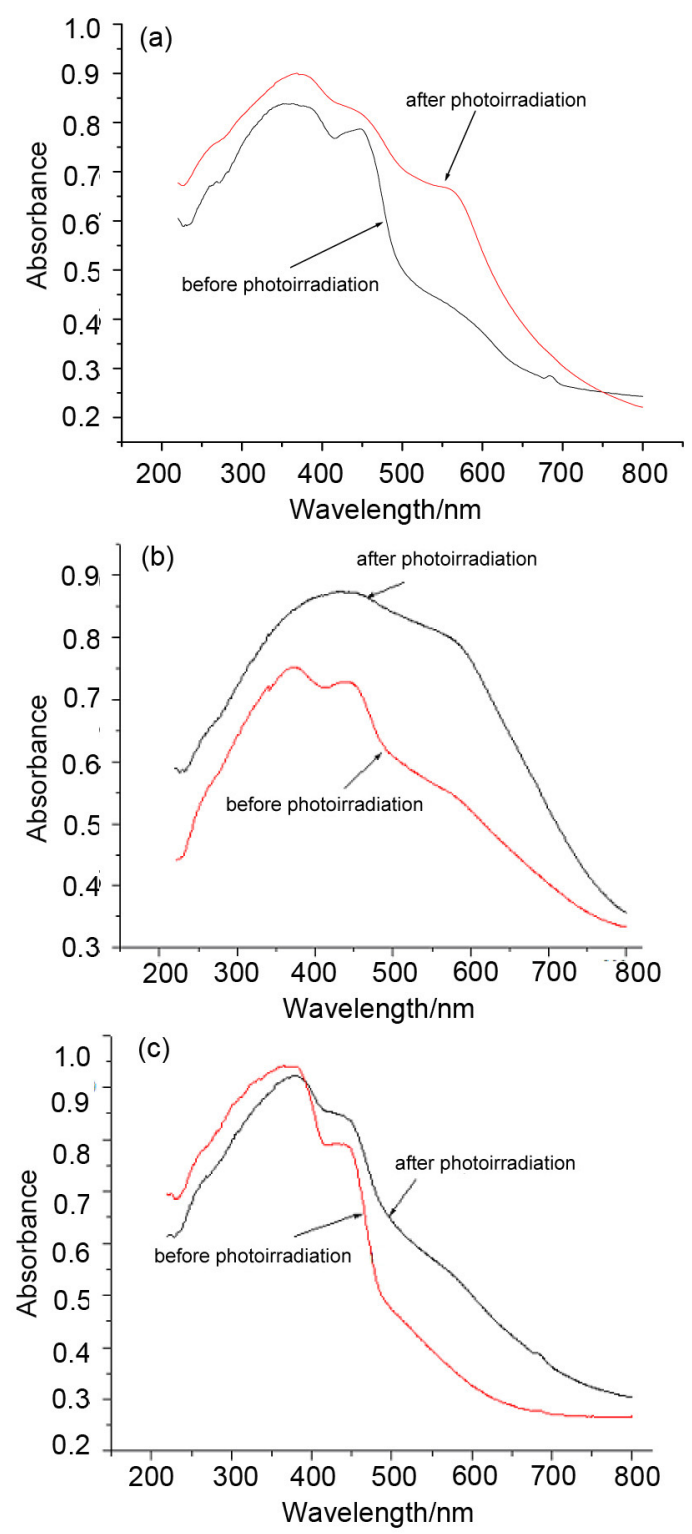

图 5 固态化合物 2 4 用高压录灯光照前后的 UV-Vis 光谱图 Figure 5 The changes of UV-Vis spectral of compound $\mathbf{2} \sim \mathbf{4}$ before and after upon irradiation with high pressure Hg lamp in the solid state

(a) 2 , (b) 3 , (c) 4

表 2 固态化合物 $2 \sim 4$ 用高压录灯光照射前后的颜色变化 Table 2 The color changes of compound 2, 3, and $\mathbf{4}$ before and after upon irradiation with high pressure $\mathrm{Hg}$ lamp in the solid state

\begin{tabular}{ccc}
\hline 化合物 & 光照前 & 光照后 \\
\hline $\mathbf{2}$ & 黄色 & 砖红色 \\
$\mathbf{3}$ & 淡黄色 & 咖啡色 \\
$\mathbf{4}$ & 土黄色 & 棕褐色 \\
\hline
\end{tabular}

对固态化合物 3 的 ESR 波谱测试结果表明，其 ESR 信号和化合物 $\mathbf{2}$ 的 ESR 信号明显不同. 固态化合物 $\mathbf{3}$ 在 没有光照的情况下，即可呈现不对称的三线态 ESR 信 号(图 7a). 以往的研究表明固态联狮满烯二酮衍生物如 


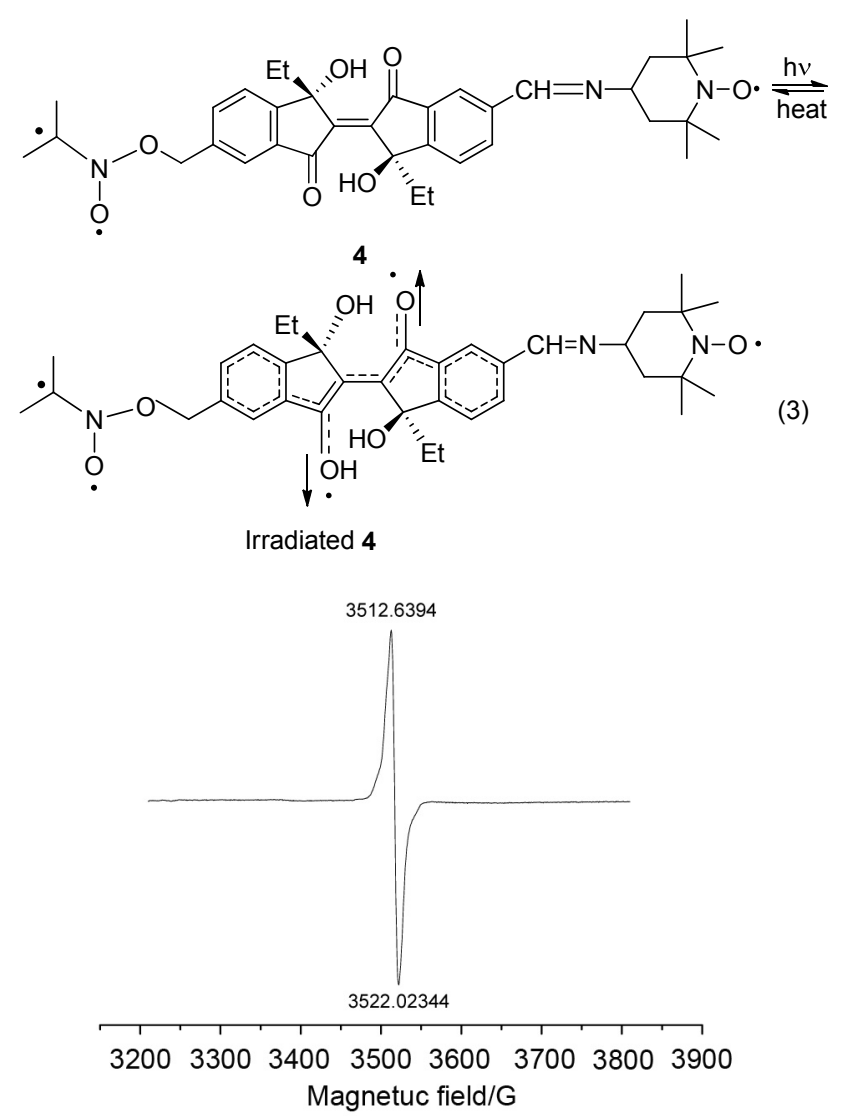

图 6 化合物 2 在室温固态条件下用高压录灯照射后的 ESR 谱图

Figure 6 ESR spectra of 2 after photoirradiation with high pressure $\mathrm{Hg}$ lamp in solid state at room temperature (work frequency, $9.858 \mathrm{GHz}$ )

果不进行光照, 不产生 ESR 信号. 而化合物 3 不进行光 照也能产生 ESR 信号, ESR 信号的自旋源来自哪里呢? 很显然来自 2-硝基丙烷自由基, 其可作为一种新的自旋 源. 固态化合物 3 光照后的 ESR 信号和其光照前比较类 似, 但光照后 ESR 信号吸收峰的位置和光照前不同, 并 在 3513.47962 处出现一个肩峰(图 7b). 为什么光照后化 合物 3 的 ESR 波谱发生变化呢? 原因是光照前化合物 3 分子中只有一个自旋中心来自 2-硝基丙烷自由基, 光照 后化合物 3 在分子中的二羰基部分产生了新的自由基, 这样光照后化合物 3 就有两种自旋中心(Scheme 8). 光 照后在化合物 3 分子内或分子间就存在两种自旋相互作 用，进而导致 ESR 波谱的变化.

在室温下, 无论把光照前的化合物 $\mathbf{3}$ 或是把光照后 的化合物 3 溶于二氯甲烷中, 测试其溶液的 ESR 波谱, 其 ESR 波谱都呈现对称的三线态模式(图 7c), 朗德因子 $g_{\text {iso }}=2.0062$, 吸收峰强度比为 $6: 6: 5$, 超精细偶合常 数: $\alpha_{\mathrm{N}}=15.7 \mathrm{G}$.

化合物 4 光照前后的 ESR 波普如图 8 所示, 光照前 化合物 4 的 $\mathrm{ESR}$ 波谱呈单线态 $\left(g=2.009, \Delta H_{\mathrm{pp}}=\right.$

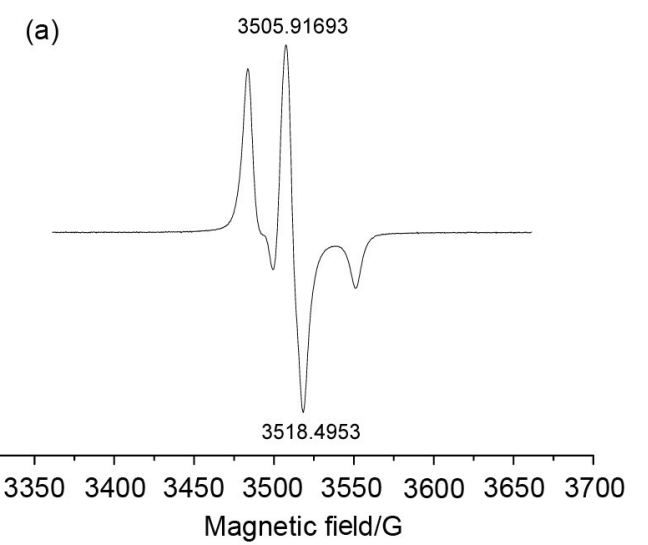

(b)

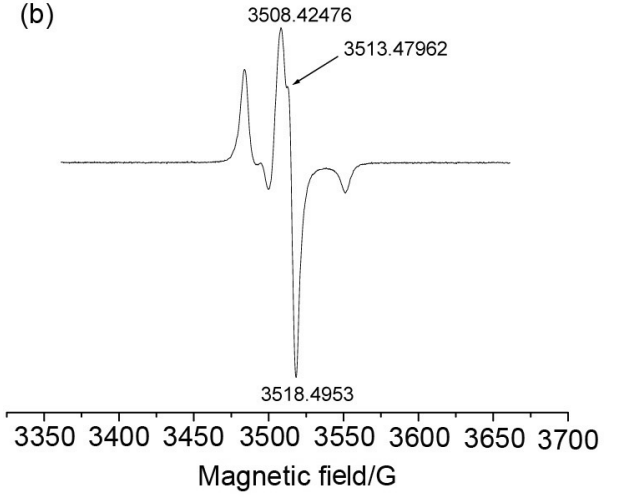

(c)

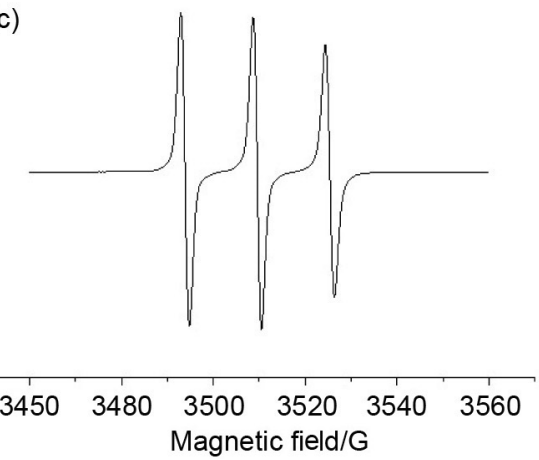

图 7 化合物 3 在室温时不同状态下的 ESR 谱 Figure 7 ESR spectrum of $\mathbf{3}$ in different states at room temperature

(a) Initial in solid state, (b) after irradiation with high pressure Hg lamp in the solid state, (c) in a dichloromethane solution (work frequency, 9.858 $\mathrm{GHz})$

27.37061) (图 8a), 而其前体化合物 3 光照前 ESR 波谱呈 现不对称的三线态，说明在化合物 4 中 TEMPO 自由基 和 2-硝基丙烷自由基相互作用形成了一个较宽的单线 态 ESR 波谱. 光照后的 ESR 波谱和光照前比较类似，但 是 $\mathrm{ESR}$ 波谱吸收峰变宽 $\left(g=2.009, \Delta H_{\mathrm{pp}}=28.93457\right)$ (图 8b). 为什么化合物 4 光照后 ESR 波谱吸收峰变宽呢? 因为单电子自旋之间的相互作用的大小，随着自旋中心 距离的靠近而增强. 光照前, 化合物 4 分子中存在两种 自旋中心，分别是 TEMPO 自由基和 2-硝基丙烷自由基， 而光照后在化合物 4 分子的二羰基部分产生了新的自由 
基，这样光照后在化合物 4 分子中就存在三种自旋中心. 随着分子中自旋中心数的增多, 导致自旋中心的距离靠 近, 单电子自旋相互作用就增强, ESR 波谱吸收峰变宽, 即久期增宽.
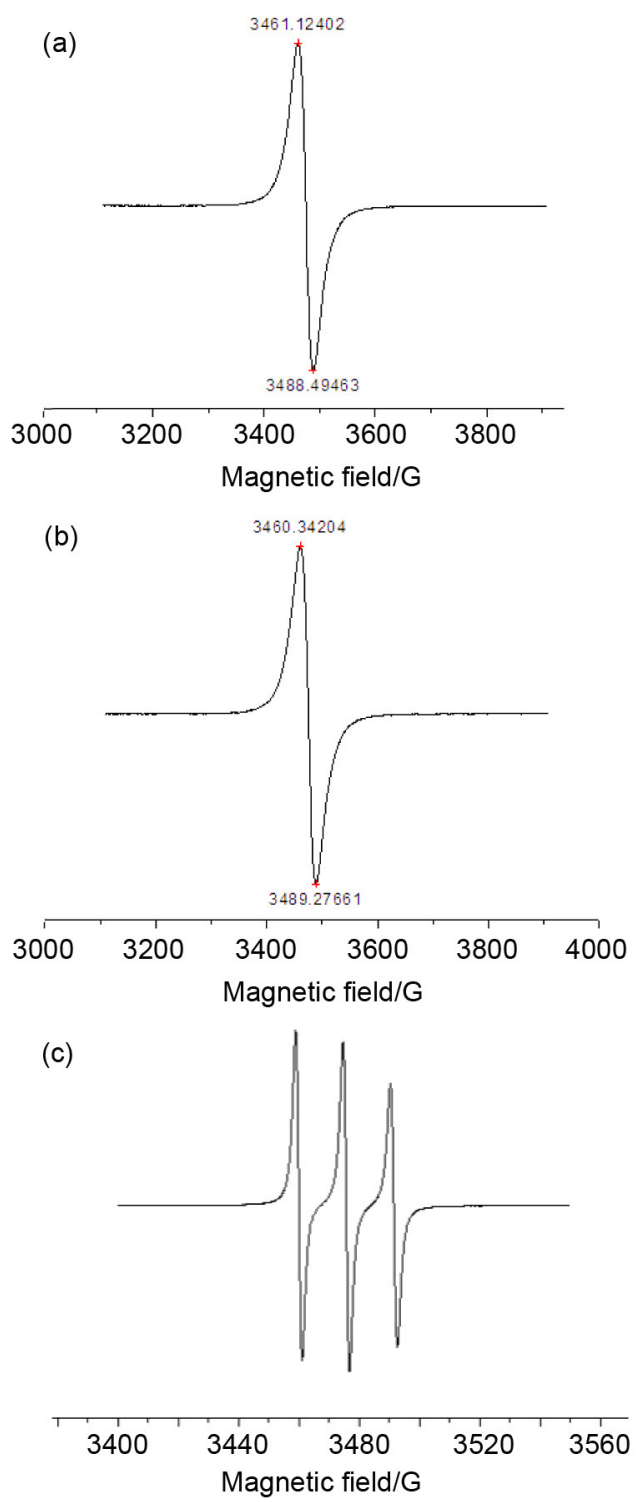

图 8 化合物 4 在室温时不同状态下的 ESR 谱图 Figure 8 SR spectrum of $\mathbf{4}$ in different states at room temperature

(a) Initial in solid state, (b) after irradiation with a high pressure Hg lamp in the solid state, (c) in a dichloromethane solution (work frequency, $9.858 \mathrm{GHz})$

在室温下, 把光照前和光照后的化合物 4 溶解到二 氯甲烷中, 其溶液的 ESR 波谱保持一致, 都呈对称的三 线态 (图 8c), 朗德因子 $g_{\text {iso }}=2.0086$, 吸收峰强度比: $4: 4: 3$, 超精细耦合常数: $\alpha_{\mathrm{N}}=15.5 \mathrm{G}$, 说明光照后化 合物 4 分子的二羰基部分产生的新自由基在溶液中淬灭 了.

\section{5 固态磁化率}

粉末状化合物 4 的变温磁化率在 MPMS XL-7 SQUID 型磁化率测试仪测试, 测试温度 2 300 K, 场强 2000 G. 测试结果见图 9. 化合物 4 光照前和光照后的变 温磁化率分别为: 光照前来自 TEMPO 自由基和 2-硝基 丙烷自由基的自旋-自旋相互作用为反铁磁相互作用, 其居里常数 $C=0.14 \mathrm{emu} \cdot \mathrm{K} \cdot \mathrm{mol}^{-1}$, 居里温度 $\theta=-0.62$ K. 光照后化合物 4 的变温磁化率曲线和光照前有很大 的不同, 其居里常数为 $C=20.6 \mathrm{emu} \cdot \mathrm{K} \cdot \mathrm{mol}^{-1}$, 居里温 度 $\theta=-238.2 \mathrm{~K}$. 实验结果清楚地表明, 光照后化合物 4 的反铁磁相互作用变得更强了, 这种增强的相互作用 来自于 TEMPO 自由基、2-硝基丙烷自由基以及光照产 生于二羰基上的自由基. 说明化合物 $\mathbf{4}$ 的磁性可受光的 调控.
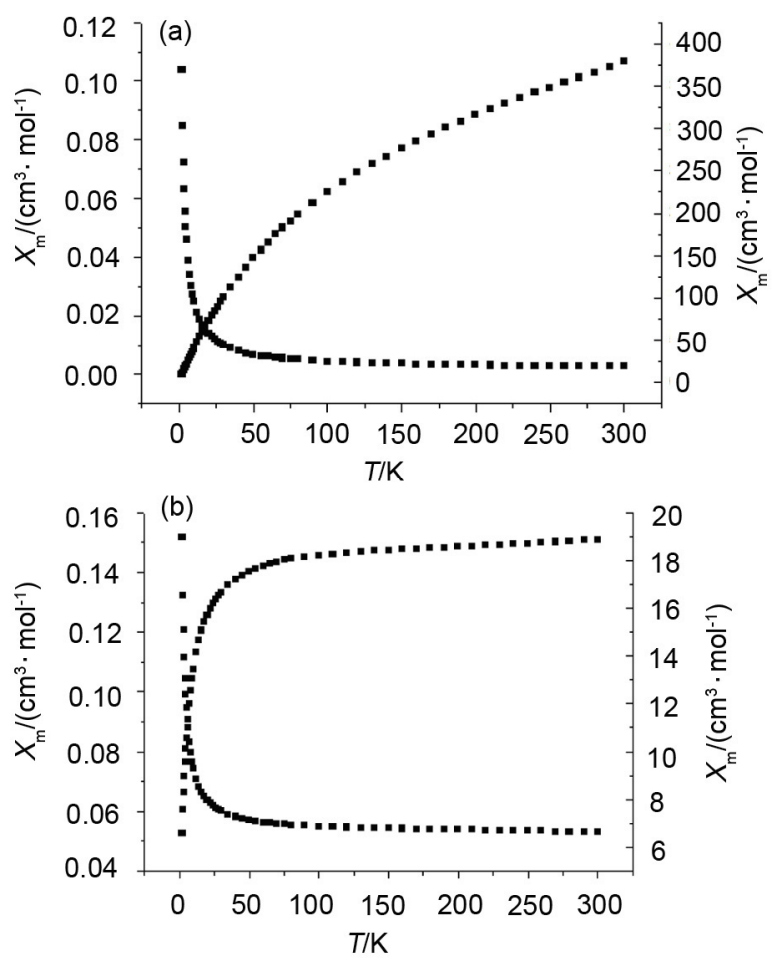

图 9 固态化合物 4 的变温磁化率 $(\chi)$ 谱图

Figure 9 Temperature dependence of the magnetic susceptibility $(\chi)$ of 4

(a) Before photoirradiation; (b) after photoirradiation

\section{2 结论}

本研究以光致变色联狮满烯二酮衍生物化合物 $\mathbf{1}$ 为 起始原料, 在合成新型光控磁性变色目标化合物的过程 中，为了合成中间体二醛 $\mathbf{X}$ ，经多种合成方法的实验， 发现化合物 1 经溴代反应，所得溴代中间体再用文献[8] 的反应方法可成功地合成醛，对中间体醛的分子结构分 析发现, 得到的是预料之外的单醛化合物 $\mathbf{3}$, 而非目的 物二醛 $\mathbf{X}$. 化合物 3 的结构经 ${ }^{1} \mathrm{H}$ NMR, MS 和元素分析 
进行了确证, 表明 2-硝基丙烷基团连接在化合物 3 苯环 的亚甲基上. 对化合物 3 的 ${ }^{1} \mathrm{H} N M R$ 波谱和 $\mathrm{ESR}$ 波谱详 细分析表明 2-硝基丙烷基团上存在自由基，提出了 2-硝 基丙烷基团上存在单电子自旋的机理. 在化合物 3 的基 础上, 稳定的 TEMPO 氮氧自由基被成功地引入化合物 3 的苯环上, 合成了新的多功能化合物 4 . 对化合物 3,4 的光致变色性质, 光照导致的磁性变化进行了研究. 实 验表明化合物 3,4 具有光致变色性质, 光照前后其 ESR 波谱发生变化. 对化合物 $\mathbf{4}$ 的变温磁化率的测试结果表 明, 光照后其反铁磁相互作用明显增强. 这些实验结果 说明化合物 3,4 光照后比光照前分子中的自旋中心增 多，导致其分子内或分子间的自旋-自旋相互作用增强. 化合物 4 的颜色和磁性可用光调控.

\section{3 实验部分}

\section{1 仪器与试剂}

熔点用 Yanagimoto MP-35 熔点仪测定(日本 Yanaco 公司), 温度计未校正; 核磁共振谱 ( ${ }^{1} \mathrm{H}$ NMR) 用 BRUKER AC-P300 核磁共振谱仪测定(德国, Bruker 公 司), 溶剂为 $\mathrm{CDCl}_{3}, \mathrm{TMS}$ 为内标; 紫外-可见光谱用 UV-2100PC 紫外-可见光谱仪测定(美国尤尼柯公司); 质谱用 Thermo Finnigan LCQ Advantage (ESI)质谱仪测 定 (美国菲尼根质谱公司); 红外波谱用 NICOLET-380-FT-IR 红外光谱仪测定(美国 Nicolet 公 司); 元素分析用 Yanaco CHN CORDER MT-3 元素分析 仪测定(日本 Yanaco 公司); 粉末 ESR 波谱用 Bruker A-320 EPR 谱仪测定(德国, Bruker 公司); 变温磁化率用 MPMS XL-7 SQUID 型磁强计测定(美国, Quantum Design 公司); 柱层析硅胶 200 300 目(青岛海洋化工厂). 所用化学试剂均为市售分析纯. 化合物 $\mathbf{1}$ 的合成见文献 [6d].

\section{2 化合物的合成}

3.2.17, $7^{\prime}$-二(一溴代甲基)-3,3'-二乙基-3,3'-二羟基$2,2^{\prime}$-二狮叉基-1, $1^{\prime}$-二酮(2)的合成

把化合物 1 (1.000 g, $2.66 \mathrm{mmol}), \mathrm{NBS}(1.000 \mathrm{~g}, 5.62$ $\mathrm{mmol}$ )和 $80 \mathrm{~mL}$ 苯加入反应瓶中, 光照下加热回流反应 $20 \mathrm{~h}$. 蒸去苯, 固体残余物过硅胶色谱柱 [洗脱液: $V($ 正已烷 $): V($ 乙醚 $)=4: 1$ ], 得黄色固体化合物 2 (0.576 g, 41\%). m.p. $187 \sim 189{ }^{\circ} \mathrm{C} ;{ }^{1} \mathrm{H}$ NMR $\left(\mathrm{CDCl}_{3}, 300\right.$ MHz) $\delta: 0.58(\mathrm{t}, J=7.4 \mathrm{~Hz}, 6 \mathrm{H}), 2.16 \sim 2.26(\mathrm{~m}, 4 \mathrm{H}), 4.56$ (s, 4H), 6.58 (s, 2H), $7.58 \sim 7.87(\mathrm{~m}, 6 \mathrm{H})$; IR (KBr) v: 3357.14, 2960.25, 2932.30, 1708.07, 1668.94, 1607.45, $1406.21,1288.82,1216.15,1154.66,1054.04 \mathrm{~cm}^{-1}$; MS (ESI) $m / z$ : $533.5[\mathrm{M}-\mathrm{H}]^{+}$. Anal. calcd for $\mathrm{C}_{24} \mathrm{H}_{22} \mathrm{O}_{4} \mathrm{Br}_{2}: \mathrm{C}$ 53.93, H 5.12; found C 53.85, H 5.19.
3.2.2 含有 2-硝基丙烷自由基和醛基的联狮满烯二 酮衍生物(3)的合成

把 $16 \mathrm{mg}(0.70 \mathrm{mmol})$ 的金属钠溶于 $25 \mathrm{~mL}$ 绝对无水 乙醇中, 然后加入 $80 \mathrm{mg}(0.90 \mathrm{mmol}) 2$ 2-硝基丙烷和 160 $\mathrm{mg}(0.30 \mathrm{mmol})$ 化合物 2 . 反应混合物在 $65 \sim 70{ }^{\circ} \mathrm{C}$, 磁 力摚拌反应 $12 \mathrm{~h}$. 蒸去乙醇, 余物过硅胶色谱柱[洗脱 液: $V($ 正己烷 $) ： V($ 乙醚 $)=4 ： 1$ ], 得淡黄色固体化合物 3 (46 mg, 32\%). m.p. $162 \sim 164{ }^{\circ} \mathrm{C} ;{ }^{1} \mathrm{H}$ NMR $\left(\mathrm{CDCl}_{3}, 300\right.$ MHz) $\delta: 0.58(\mathrm{t}, J=3.3 \mathrm{~Hz}, 6 \mathrm{H}), 1.66(\mathrm{~d}, J=14.1 \mathrm{~Hz}, 6 \mathrm{H})$, $2.17 \sim 2.30(\mathrm{~m}, 4 \mathrm{H}), 3.33 \sim 3.46(\mathrm{~m}, 2 \mathrm{H}), 6.45(\mathrm{~s}, 1 \mathrm{H})$, $6.69(\mathrm{~s}, 1 \mathrm{H}), 7.31 \sim 7.34(\mathrm{~m}, 1 \mathrm{H}), 7.57(\mathrm{~s}, 1 \mathrm{H}), 7.83(\mathrm{~d}$, $J=7.8 \mathrm{~Hz}, 1 \mathrm{H}), 8.01(\mathrm{~s}, 1 \mathrm{H}), 8.36(\mathrm{t}, J=2.9 \mathrm{~Hz}, 2 \mathrm{H})$, 10.16 (s, 1H); IR (KBr) v: 3385.09, 2960.25, 2937.89, $2361.75,1702.48,1668.94,1607.45,1540.37,1400.62$, 1266.46, 1154.66, $1098.76 \mathrm{~cm}^{-1}$; MS (ESI) $\mathrm{m} / z: 476.4$ $[\mathrm{M}-\mathrm{H}]^{+}$. Anal. calcd for $\mathrm{C}_{27} \mathrm{H}_{27} \mathrm{O}_{7} \mathrm{~N}: \mathrm{C} 67.92, \mathrm{H} 5.66, \mathrm{~N}$ 2.94; found C 67.86, H 5.61, N 2.89.

3.2.3 含有2-硝基丙烷自由基和 TEMPO 自由基的联 䒢满烯二酮衍生物 4 的合成

把 $100 \mathrm{mg}(0.159 \mathrm{mmol})$ 化合物 3 溶于 $10 \mathrm{~mL}$ 的苯中, 然后向其中加入 $28 \mathrm{mg}(0.16 \mathrm{mmol})$ 4-amino-TEMPO, 再滴加两滴乙酸, 氮气保护下, 控温 $45{ }^{\circ} \mathrm{C}$, 磁力搅拌 反应 $10 \mathrm{~h}$, 旋蒸除去苯, 余物用 $V($ 正己烷 $): V($ 苯 $)=$ $1: 1$ 的混合液重结晶, 得土黄色固体化合物 $4(30 \mathrm{mg}$, $23 \%$ ). m.p. $176 \sim 178{ }^{\circ} \mathrm{C}$; IR (KBr) v: 3373.73, 2967.28, 2921.66, 2361.75, 1706.45, 1669.12, 1602.77, 1540.55, $1457.60,1345.62,1262.67,1167.28,1096.77 \mathrm{~cm}^{-1}$. HRMS (ESI) calcd for $\mathrm{C}_{36} \mathrm{H}_{44} \mathrm{~N}_{3} \mathrm{O}_{7}$ 631.3252, found 631.3250. Anal. calcd for $\mathrm{C}_{36} \mathrm{H}_{44} \mathrm{~N}_{3} \mathrm{O}_{7}: \mathrm{C} 68.55, \mathrm{H} 7.03, \mathrm{~N}$ 6.66; found C 68.50, H 7.08, N 6.61.

辅助材料(supporting information) 化合物 1 的分子结 构单晶衍射图，化合物 $\mathbf{2}, \mathbf{3}, \mathbf{4}$ 的 $g$ 值计算，化合物 1 的 ${ }^{1} \mathrm{H}$ NMR 谱, $\Delta H_{\mathrm{pp}}$ 的解释，化合物 $\mathbf{2} \sim \mathbf{4}$ 的 ESR 波谱的测 试条件. 这些材料可以免费从本刊网站 (http://siocjournal.cn)上下载.

\section{References}

[1] (a) Scott, K. S.; Dennis, A. D. J. Phys. Chem. 1993, 97, 13273. (b) Miller, J. S.; Epstein, A. J. Angew. Chem., Int Ed. Engl. 1994, 33,385 .

(c) Zhou. B.; Idobata, Y.; Kobayashi, A.; Cui, H. B.; Kato, R.; Takagi, R.; Miyagawa, K.; Kanoda, K.; Kobayashi, H. J. Am. Chem. Soc. 2012, 134, 12724.

(d) Li, Z. X.; Yang, Q.; Li, L. C.; Hu, T. L.; Bu, X. H. Acta Chim. Sinica 2013, 71, 755 (in Chinese).

(李佐习, 杨乾, 李立存, 卜显和, 化学学报, 2013, 71, 755.)

(e) Barch, M.; Okada, S.; Bartelle, B. B.; Jasanoff, A. J. Am. Chem. 
Soc. 2014, 136, 12516.

(f) Aaron. M.; Stephen, M. W.; Joanne, W. L.; Craig, M. R.; Abdeljalil, A.; Paul, A. D.; Richard, T. O. J. Am. Chem. Soc. 2015, 137, 1044.

(g) Ren, W.; Zheng, L. M. Acta Chim. Sinica 2015, 73, 1091 (in Chinese).

(任旻, 郑丽敏, 化学学报, 2015, 73, 1091.)

[2] (a) Nakatsuji, S.; Anzai, H. J. Mater. Chem. 1997, 7, 2161.

(b) Franciso, M. R.; Raymond, Z.; Michel, M. B.; Yves, P.; Eric, R.; Jacques. S.; Bernard, D.; Andre, G.; Carley, P. J. Am. Chem. Soc. 2000, 122, 1298.

(c) Robin, G. H.; Martin, T. L.; Lars, O.; John, F. R.; Laurence, K. T.; Xu, Z. Q. J. Am. Chem. Soc. 2001, 123, 7154.

(d) Pascale, W.; Jacques, L. M.; Vladimira, V.; Philippe, T. J. Org. Chem. 2003, 68, 8025 .

(e) Raymond, Z.; Christophe, S.; Henrike, H.; Frank, H.; Kohler, P. T.; Nicolaser, C.; Mohamed, S.; Claude, L. J. Am. Chem. Soc. 2004, 126, 12604.

(f) Li, J.; Hironari, I.; Keiichi, K.; Takaumi, M.; Brian, K. B.; Masahiro, Y.; Tadahiro, K. J. Am. Chem. Soc. 2013, 135, 651.

[3] (a) Irie, M. Chem. Rev. 2000, 100, 1685.

(b) Delaire, J. A.; Nakatani, K. Chem. Rev. 2000, 100, 1817.

(c) Idowu, M.; Nyokong, T. J. Photochem. Photobiol., A 2007, 188, 200.

(d) Chen, Q.; Feng, Y.; Zhang, D. Q.; Zhang, G. X.; Fan, Q. H.; Sun, S. N.; Zhu, D. B. Adv. Funct. Mater. 2010, 20, 36.

(e) Wang, Z. Q.; Xiao, Y.; Jin, T. F.; Wang, S. R.; Li, X. G. Acta Chim. Sinica 2014, 72, 731 (in Chinese).

(王志强, 肖殷, 金会义, 谈廷凤, 王世荣, 李祥高, 化学学报, 2014, 72, 731.)

(f) Yang, Y.; Zhang, G. X.; Yu, C. M.; He, C.; Wang, J. G.; Chen, X.; Yao, J. J.; Liu, Z. T.; Zhang, D. Q. Chem. Commun. 2014, 50, 9939. (g) Chan, J. C.; Lam, W. H.; Yam, V. W. J. Am. Chem. Soc. 2014, 136, 16994.

[4] (a) Li, X.; Han, J.; Pang, M. L.; Chen. Y.; Zhang, J. X.; Ma, H.; He,
Z. J.; Meng, J. B. Tetrahedron Lett. 2007, 48, 6044.

(b) Chen, Y.; Pang, M. L.; Cheng, K. G.; Wang, Y.; Han, J.; Meng, J. B. J. Photochem. Photobiol. A 2008, 194, 122.

(c) Chen, Y.; Pang, M. L.; Cheng, K. G.; Wang, Y.; Han, J.; Meng, J. B. Acta Chim. Sinica 2008, 66, 1091 (in Chinese).

(陈勇, 庞美丽, 程凯歌, 王英, 韩杰, 孟继本, 化学学报, 2008, 66, 1091.)

(d) Chen, Y.; Pang, M. L.; Cheng, K. G.; Wang, Y. Chin. J. Chem. 2012, 30, 1759.

(e) Cai, L. Z.; Chen, Q. S.; Zhang, C. J.; Li, P. X.; Wang, M. S.; Guo, G. C. J. Am. Chem. Soc. 2015, 135, 10882.

[5] (a) Matsuda, K.; Matsuo, M.; Irie, M. J. Org. Chem. 2001, 66, 8799 .

(b) Matsuda, K.; Matsuo, M.; Mizoguti, S.; Higashiguchi, K.; Irie, M. J. Phys. Chem. B 2002, 106, 11218.

(c) Tanifuji, N.; Irie, M.; Matsuda, K. J. Am. Chem. Soc. 2005, 127, 13344.

(d) Matsuda, K. Bull. Chem. Soc. Jpn. 2005, 78, 383.

[6] (a) Xu, L. L.; Sygiyama, T.; Meng, J. B.; Matsuua, T. Chem. Commun. 2002, 2328.

(b) Li. X.; Song, Z. Y.; Chen, Y.; Meng, J. B. J. Mol. Struct. 2005, $748,161$.

(c) Li, X.; Xu, L. L.; Han, J.; Pang, M. L.; Ma, H.; Meng, J. B. Tetrahedron 2005, 61, 5373.

(d) Chen, Y.; Pang. M. L.; Cheng, K. G.; Wang, Y.; Han, J.; He, Z. J.; Meng, J. B. Tetrahedron 2007, 63, 4319.

(e) Chen, Y.; Pang, M. L.; Cheng, K. G.; Wang, Y.; Han, J.; Meng, J. B. Chin. J. Chem. 2010, 28, 1240.

[7] (a) Walton, A. F.; Tipson, R. S.; Cretche, L. H. J. Am. Chem. Soc. 1945, 67, 1501.

(b) Marvel, C. S.; Saunders, J. H.; Overberc, C. G. J. Am. Chem. Soc. 1946, 68, 1085.

(c) Vlttas, I.; Harrison, I. T.; Tokes, L.; Fried, J. H.; Cros, A. D. J. Org. Chem. 1968, 33, 4176.

[8] Hass, H. B.; Bender, M. L. J. Am. Chem. Soc. 1949, 71, 1767.

(Qin, X.) 